\title{
HETEROCONTROLE DA FLUORETAÇÃO DA ÁGUA DE ABASTECIMENTO PÚBLICO DA 15a REgIONAL dE SAÚdE do PARANÁ
}

\author{
Heterocontrol of fluoridation of public water supply \\ 15 $5^{\text {th }}$ Health Region (state of Paraná, Brazil)
}

Tânia Harumi Uchida ${ }^{1}$, Jaime Aparecido $\mathrm{Cury}^{2}$, Cínthia Pereira Machado Tabchoury ${ }^{3}$, Raquel Sano Suga Terada ${ }^{4}$, Mitsue Fujimaki ${ }^{5}$

1. Doutoranda do Programa de Pós-Graduação em Odontologia Integrada da Universidade Estadual de Maringá - UEM, Maringá, Paraná, Brasil. ORCID: http://orcid.org/0000-0001-8170-1092.

2. Doutor e Professor Titular da Faculdade de Odontologia de Piracicaba - FOP/ UNICAMP, Piracicaba, São Paulo, Brasil. ORCID: http://orcid.org/0000-0003-1046-5605.

3. Doutora e Professora Associada da Faculdade de Odontologia de Piracicaba - FOP/ UNICAMP. Piracicaba, São Paulo, Brasil. ORCID: http://orcid.org/0000-0002-7660-5685.

4. Doutora e Professora Associada da Universidade Estadual de Maringá - UEM, Maringá, Paraná, Brasil. ORCID: http://orcid.org/0000-0003-1344-9870.

5. Doutora e Professora Associada da Universidade Estadual de Maringá - UEM, Maringá, Paraná, Brasil. ORCID: http://orcid.org/0000-0002-7824-3868.

CONTATO: Mitsue Fujimaki | Rua Luiz Gama, 144 | Apto 1601 | Centro | Maringá | Paraná | Brasil | CEP 87014-110 | Cel: (44) 99111-6464 | E-mail: mfujimaki@uem.br

COMO CITAR Uchida TH, Cury JA, Tabchoury COM, Tereda RSS, Fujimaki M. Heterocontrole da fluoretação da água de abastecimento público da 15a Regional de Saúde do Paraná. R. Saúde Públ. 2018 Jul.,1(1):59-67.

RESUMO Objetivou-se conhecer o processo de fluoretação das águas de abastecimento público e realizar o heterocontrole do fluoreto nas águas dos 30 municípios que compõem a 15a Regional de Saúde do Paraná. Um questionário contendo dados relativos à fluoretação dos municípios foi aplicado e cinco coletas de água em Unidades 
Básicas de Saúde ou poços artesianos foram realizadas em todos esses municípios, no período de 12 meses. Dos 30 municípios que compõem esta regional, em 77\% dos municípios a água utilizada é proveniente de poços, $77 \%$ relatam ter água fluoretada e 40\% dos municípios realizaram levantamento epidemiológico para fluorose dental. Das 335 amostras de água, 79,5\% foram classificadas como máximo benefício contra cárie e mínimo risco para fluorose dentária. Conclui-se que apesar da maioria dos municípios pesquisados realizarem a fluoretação das águas de abastecimento, o heterocontrole não é prática comum nos mesmos.

PALAVRAS-CHAVE: Fluoretação. Fluoretos. Abastecimento de água.

\begin{abstract}
The objective of this study was to know the process of fluoridation of the public water supply, and to perform the heterocontrol of fluoride in the waters of 30 municipalities of the 15th Regional Department of Health of Paraná. A questionnaire containing data on fluoridation in the municipalities was applied, and five water collections in Basic Health Units, or in artesian wells, were carried out in all these municipalities, in a period of 12 months. Of the 30 municipalities composing this regional department, $77 \%$ use water from wells, $77 \%$ reported having fluoridated water, and $40 \%$ carried out an epidemiological survey for dental fluorosis. Of the 335 water samples, $79.5 \%$ were classified as the maximum benefit against dental decays, and minimal risk for dental fluorosis. It is concluded that most of the cities surveyed perform fluoridation of water supply, but heterocontrol is not a common practice.
\end{abstract}

KEYWORDS: Fluoridation. Fluorides. Water supply.

\title{
INTRODUÇÃO
}

A

cárie dentária, em termos de saúde pública, é o principal problema de saúde bucal'. A adição de fluoreto $(F)$ na água de abastecimento público pode, de forma isolada, reduzir as chances de uma pessoa ter cárie em até 65\% e, além disso, é um método recomendado por mais de 150 organizações de ciências e saúde, dentre elas, a Organização Mundial da Saúde, a Federação Dentária Internacional, a Associação Internacional de Pesquisa em Odontologia, a Organização Pan-Americana de Saúde e a American Dental Association². A fluoretação das águas de abastecimento público é um método coletivo e representa umas das principais e mais importantes medidas de saúde pública, podendo ser considerada como uma medida de controle de cárie dentária efetiva e de ótima relação custo-benefício ${ }^{3}$.

Entretanto, para que a população se beneficie dos efeitos dessa importante medida, faz-se necessário que os teores de $\mathrm{F}$ adicionados à água de abastecimento público estejam dentro dos níveis recomendados ${ }^{4}$. É necessária a existência de mecanismos que viabilizem e controlem a adequada concentração de $F$ na água, para que a medida exerça o maior impacto possível na prevenção da cárie, sem aumentar a prevalência de fluorose dental $^{5}$. Os niveis de $\mathrm{F}$ devem permanecer dentro de faixas adequadas e por longos períodos de tempo para ter seus efeitos desejados ${ }^{6}$, reforçando a importância de se ter um sistema de vigilância para a manutenção desses níveis. Um sistema de vigilância permanente da fluoretação deve ser realizado pela própria companhia de abastecimento de água (controle operacional) ou por meio de um 
mecanismo externo conhecido como heterocontrole, o qual compreende o monitoramento dos teores de $F$ nas águas de abastecimento por uma instituição ou órgão diferente daquele responsável pela fluoretação?

No Brasil, a primeira cidade a implantar a fluoretação das águas em seu sistema de abastecimento foi a cidade de Baixo Guandu, no Estado do Espírito Santo, em 19538. Apesar da fluoretação ser obrigatória no Brasil desde 1974 (lei $\mathrm{n}^{\circ}$. 6050, de 24 de maio de 1974)1', em localidades onde exista Estação de Tratamento de Água (ETA), vários municípios ainda não adicionam $F$ nas suas águas de abastecimento público. A portaria do Ministério da Saúde $n^{\circ}$. 635, de 25 de dezembro de 1975, aprova e determina normas e padrões a serem seguidos para a correta fluoretação das águas, desde a concentração a ser utilizada, de acordo com as médias das temperaturas máximas anuais de cada região ${ }^{2.8}$. Em 2011, uma nova classificação para a concentração de $\mathrm{F}$ nas águas de abastecimento público (Centro Colaborador do Ministério da Saúde para Vigilância em Saúde Bucal da Universidade de São Paulo - CECOL/USP) foi construída coletivamente e consensuada pela comunidade acadêmica, especialistas e produtores de água. Esta nova classificação da concentração de $\mathrm{F}$ é baseada em benefício anticárie e risco de fluorose ${ }^{9}$. Por isso, estudos de heterocontrole da fluoretação tornam-se importantes.

Até o momento, pouco se sabe sobre o heterocontrole do processo de fluoretação das águas de abastecimento público na $15^{\mathrm{a}}$ Regional de Saúde do Paraná. Desta maneira, o objetivo deste trabalho foi conhecer o processo de fluoretação das águas de abastecimento público e realizar o heterocontrole do $\mathrm{F}$ nas águas dos 30 municípios que compõem a $15^{a}$ Regional de Saúde do Paraná. Exceto em Maringá, nenhum estudo de heterocontrole foi feito nesses municípios e poucos são os trabalhos que utilizam essa nova classificação, proposta pelo CECOL/USP, considerando o máximo benefício na prevenção da cárie dentária e mínimo risco de desenvolvimento da fluorose dental.

\section{METODOLOGIA}

\section{DELINEAMENTO DA PESQUISA}

Trata-se de um estudo descritivo, exploratório e longitudinal. Buscou-se avaliar o processo de fluoretação das águas de abastecimento dos municípios pertencentes à $15^{\mathrm{a}}$ Regional de Saúde do Paraná, por meio de um questionário entregue aos coordenadores de saúde bucal de cada município. Para a realização do heterocontrole da fluoretação das águas de abastecimento, foram realizadas coletas trimestrais de amostras de água, provenientes de pontos específicos, como as Unidades Básicas de Saúde (UBSs), poços artesianos ou minas em um período de 12 meses.

\section{LOCAL DO ESTUDO}

O estado do Paraná possui a Secretaria da Saúde do Paraná (SESA/PR), dividida em 22 regionais de saúde, e o presente estudo foi realizado na $15^{\mathrm{a}}$ Regional de Saúde. Esta regional abrange 30 municípios da região Noroeste (Ângulo, Astorga, Atalaia, Colorado, Doutor Camargo, Floraí, Floresta, Flórida, Iguaraçu, Itaguajé, Itambé, Ivatuba, Lobato, Mandaguaçu, Mandaguari, Marialva, Maringá, Munhoz de Mello, Nossa Senhora das Graças, Nova Esperança, Ourizona, Paiçandu, Paranacity, Presidente Castelo Branco, Santa Fé, Santa Inês, Santo Inácio, São Jorge do Ivaí, Sarandi e Uniflor), com sede localizada no município de Maringá. Desses 30 municípios, 23 deles possuem fluoretação das águas de abastecimento, sendo eles: Astorga, Atalaia, Doutor Camargo, Floraí, Floresta, Itaguajé, Itambé, Ivatuba, Lobato, Mandaguaçu, Mandaguari, Maringá, Munhoz de Mello, Nova Esperança, Ourizona, Paiçandu, Paranacity, Presidente Castelo Branco, Santa Fé, Santa Inês, Santo Inácio, São Jorge do Ivaí, e Uniflor. Sete municípios não adicionam o $\mathrm{F}$ na água de abastecimento público: Ângulo, Colorado, Flórida, Iguaraçu, Marialva, Nossa Senhora das Graças e Sarandi. 


\section{AVALIAÇÃO DA FLUORETAÇÃO DA ÁGUA DE ABASTECIMENTO DOS MUNICIPIOS}

Foram entregues pessoalmente questionários aos coordenadores de saúde bucal dos municípios durante uma reunião realizada pela coordenação de saúde bucal da $15^{\text {a }}$ Regional de Saúde do Paraná, na qual foi explicada a importância do heterocontrole e os benefícios do uso do F. Quando necessário, os mesmos foram reenviados por e-mail. 0 questionário continha questões que abordavam os seguintes assuntos: fonte de captação da água de abastecimento, presença de $\mathrm{F}$ na água de abastecimento, empresa responsável pelo tratamento da água, frequência com que é feito o controle da concentração de $\mathrm{F}$ e realização de algum levantamento epidemiológico de cárie ou fluorose dentária. A apresentação dos resultados foi realizada de forma descritiva.

\section{COLETA DAS AMOSTRAS DE ÁGUA}

Dos 23 municípios que adicionam $F$ na água de abastecimento público, sete municípios (Astorga, Mandaguaçu, Mandaguari, Maringá, Nova Esperança, Paiçandu e Paranacity) tiveram as amostras de água coletadas das UBSs, 1 município (Munhoz de Mello) teve água coletada de minas e em 15 municípios (Atalaia, Doutor Camargo, Floraí, Floresta, Itaguajé, Itambé, Ivatuba, Lobato, Ourizona, Presidente Castelo Branco, Santa Fé, Santa Inês, Santo Inácio, São Jorge do Ivaí, e Uniflor) a água foi coletada diretamente de poços, onde ocorria o tratamento e fluoretação. Considerando a diversidade de sistemas de tratamento de água nos municípios da $15^{\mathrm{a}}$ Regional de Saúde, o número de pontos de coleta não seguiu o mesmo padrão para todos os municípios.

Como a adesão dos municípios ocorreu paulatinamente, devido à dificuldade dos coordenadores de saúde bucal dos municípios da 15a Regional do Paraná aderirem ao projeto do heterocontrole, não foi possivel realizar as coletas de amostras de água de todos os municípios ao mesmo tempo. Assim, o período de coleta das amostras foi de dezembro de 2010 a fevereiro de 2013.
Para as coletas, foram utilizados frascos plásticos, previamente etiquetados e identificados. Ao responsável pela coleta era solicitado que a água fosse oriunda da torneira situada o mais próximo da entrada de água da rua, preferencialmente junto ao hidrômetro. Esta torneira era deixada aberta por trinta segundos, os frascos lavados três vezes e a água então era coletada para análise.

Nos 7 municípios que não apresentaram fluoretação das águas de abastecimento foi realizada somente a primeira coleta proveniente de UBS, poço ou mina, para comprovação.

\section{ANÁLISE DAS AMOSTRAS}

A verificação do teor de $F$ na água foi feita pelo Laboratório de Bioquímica Oral da Faculdade de Odontologia de Piracicaba (FOP/UNICAMP). A concentração de $F$ das amostras foi determinada com eletrodo íon específico (Orion 96-09) acoplado a um analisador de íons (ORIONSTAR A 214). 0 aparelho foi calibrado com soluções padrões em concentrações de $F$ variando de 0,25 a 2,00 $\mu \mathrm{g}$ $\mathrm{F} / \mathrm{mL}$, adicionando-se o mesmo volume de TISAB II. As leituras dos padrões foram realizadas em triplicata para a construção da curva de calibração. Em seguida, as amostras foram analisadas em duplicata e os resultados obtidos foram calculados e expressos em ppm F.

\section{CLASSIFICAÇÃO DAS AMOSTRAS}

Todas as amostras avaliadas foram classificadas de acordo com o documento do consenso técnico sobre a Classificação de Águas de Abastecimento Público segundo o Teor de Flúor ${ }^{9}$. Os municípios pertencentes à $15^{\text {a }}$ Regional de Saúde do Paraná estão localizados na região Noroeste do estado do Paraná, onde as médias das temperaturas máximas se situam entre 26,3 e $32,5^{\circ} \mathrm{C}$. Portanto, a concentração de $\mathrm{F}$ que oferece a melhor combinação benefício-risco varia de 0,55 a 0,84 ppm de F, na qual o benefício na prevenção da cárie é máximo e o risco de produzir fluorose é mínimo. 


\section{RESULTADOS}

Todos os 30 questionários enviados aos municípios foram respondidos. Os resultados estão resumidos na Tabela 1.

Tabela 1. Aspectos relevantes obtidos mediante questionário realizado com os Coordenadores de Saúde Bucal da 15a Regional de Saúde do Paraná.

QUESTIONAMENTOS

1. Fonte de captação da água de abastecimento

RESPOSTAS

- 77\% (n=23) dos municípios - captação de poços

- $10 \%(n=3)$ dos municípios - mistura com água captada de poços e de rio.

- $10 \%(n=3)$ - mistura de água captada de poço e mina.

- $3 \%(n=1)$ - captação de rio.

2. Fluoretação na água de abastecimento

- $77 \%(n=23)$ dos municípios apresentam a fluoretação das águas de abastecimento, realizada pela empresa responsável pelo tratamento da água.

- $24 \%(n=7)$ não apresentam a fluoretação das águas de abastecimento.

3. Empresa responsável pelo tratamento da água

- 63\% (n=19) dos municípios - SANEPAR.

- 23\% ( $n=7)$ - Sistema Municipal de Água e Esgoto (SAMAE).

- $14 \%(n=4)$ - serviço é terceirizado pelas prefeituras.

4. Frequência com que é feito o controle da concentração de $F$

- Dos 23 municípios que apresentam água fluoretada:

- 34,8\% ( $n=8)$ relataram fazer diariamente o controle do teor de F pela companhia de abastecimento;

- 60,85\% (n=14) relataram não haver uma frequência estabelecida;

- 4,35\% (n=1) alegou realizar heterocontrole da fluoretação da água de abastecimento.

5. Realização de algum levantamento

- $40 \%(n=12)$ dos municípios declararam terem feito algum tipo de levantamento; epidemiológico de cárie ou fluorose dentária

- 50\% ( $n=15)$ nunca fizeram e

- $10 \%(n=3)$ não souberam responder.
Foram avaliadas 335 amostras de água que passaram pelo processo de fluoretação. A Tabela 2 apresenta a porcentagem do total de amostras de água em cada faixa de concentração de $\mathrm{F}$, de acordo com a nova proposta de classificação baseada em benefício na prevenção da cárie e risco de fluorose ${ }^{9}$.

\section{DISCUSSÃO}

A fluoretação das águas de abastecimento público tornou-se, ao longo do século $X X$, uma das ações de maior impacto na prevenção da cárie dentária, desde que ocorra uma manutenção adequada e permanente dos níveis de $F^{10,11,12}$. Para garantir a eficácia preventiva da fluoretação é indispensável o controle operacional nas estações de tratamento de água e o seu monitoramento, que em termos da Vigilância em Saúde é conhecido como heterocontrole ${ }^{13}$. O heterocontrole é uma ação de vigilância em saúde pública empregado para garantir a manutenção e concentração de $F$ presentes na água, visando o máximo benefício da prevenção da cárie dentária com o mínimo risco 
Tabela 2. Porcentagem do total de amostras de água, classificadas de acordo com as faixas de concentração de $\mathrm{F}$ e seus benefício e risco estimados $(N=335)$. Para localidades em que as médias das temperaturas máximas se situam entre $26,3^{\circ}$ e $32,50 \mathrm{C}$

\begin{tabular}{|c|c|c|c|c|c|c|c|c|}
\hline \multirow{2}{*}{$\begin{array}{l}\text { Faixa de População } \\
\text { (mil habitantes) }\end{array}$} & \multirow{2}{*}{ Municípios } & \multirow{2}{*}{$\begin{array}{l}\text { Amostras } \\
\text { coletadas (n) }\end{array}$} & \multicolumn{5}{|c|}{ ppm F (\%) } & \multirow[b]{2}{*}{$>1,45$} \\
\hline & & & $\begin{array}{c}0,00 \\
a \\
0,44\end{array}$ & $\begin{array}{c}0,45 \\
a \\
0,54\end{array}$ & $\begin{array}{c}0,55 \\
a \\
0,84^{*}\end{array}$ & $\begin{array}{c}0,85 \\
a \\
1,14\end{array}$ & $\begin{array}{c}1,15 \\
a \\
1,44\end{array}$ & \\
\hline \multirow{15}{*}{0 I--- 10} & 1 & 5 & 1 & - & 4 & - & - & - \\
\hline & 2 & 5 & - & - & 5 & - & - & - \\
\hline & 3 & 5 & - & - & 5 & - & - & - \\
\hline & 4 & 5 & - & - & 5 & - & - & - \\
\hline & 5 & 5 & - & - & 5 & - & - & - \\
\hline & 6 & 5 & - & - & 5 & - & - & - \\
\hline & 7 & 5 & - & - & 5 & - & - & - \\
\hline & 8 & 5 & 5 & - & - & - & - & - \\
\hline & 9 & 5 & 3 & - & 9 & 3 & - & - \\
\hline & 10 & 5 & - & 2 & 3 & - & - & - \\
\hline & 11 & 5 & - & - & 4 & 1 & - & - \\
\hline & 12 & 5 & - & - & 5 & - & - & - \\
\hline & 13 & 5 & - & - & 5 & - & - & - \\
\hline & 14 & 5 & 2 & - & 2 & 1 & - & - \\
\hline & 15 & 5 & - & - & 2 & 3 & - & - \\
\hline \multirow{3}{*}{10 I--- 20} & 16 & 10 & 2 & 2 & 6 & - & - & - \\
\hline & 17 & 10 & - & 1 & 7 & 2 & - & - \\
\hline & 18 & 5 & - & - & 5 & - & - & - \\
\hline \multirow{4}{*}{20 I--- 50} & 19 & 25 & - & - & 18 & 7 & - & - \\
\hline & 20 & 30 & - & - & 28 & 2 & - & - \\
\hline & 21 & 10 & - & - & 8 & 2 & - & - \\
\hline & 22 & 30 & - & - & 30 & - & - & - \\
\hline \multirow[t]{2}{*}{100 I--- 500} & 23 & 130 & 5 & - & 100 & 25 & - & - \\
\hline & Total & 335 & 18 & 5 & 266 & 46 & - & - \\
\hline
\end{tabular}

Fonte: Elaborado pelos autores (2018)

de fluorose ${ }^{14}$. Resultados de revisões sistemáticas comprovam o efeito benéfico e a segurança do $F$ quando em concentração adequada na água de abastecimento ${ }^{15,16}$. Embora o Brasil seja o segundo maior sistema de fluoretação de águas de abastecimento público de todo o mundo ${ }^{17}$, o controle operacional sobre o teor de $F$ é insuficiente. O controle operacional deve ser exercido regularmente pelo responsável pelo Sistema de Abastecimento de Água de forma a assegurar a manutenção na concentração de $\mathrm{F}$ e a potabilidade da água para consumo humano ${ }^{18}$.
Assim, existe a necessidade de desenvolver mecanismos de controle da fluoretação8, onde o heterocontrole possa ser uma política e deixar de ser uma iniciativa de órgãos, como as instituições de ensino.

Com o intuito de institucionalizar o heterocontrole como uma política, o CECOL/ USP, dentre as atividades de vigilância da saúde bucal, tem dado ênfase aos aspectos relacionados com a vigilância da fluoretação das águas de abastecimento público e temas associados. Dessa maneira, o CECOL/USP apresentou um documento 
com sugestões na Consulta Pública sobre a Portaria MS 2.914, de 12/12/2011 ${ }^{19}$. O documento indica a necessidade de que as análises da concentração de F na água sejam feitas conforme as necessidades de cada região de saúde e de rever a Portaria $635 / 75$ por se encontrar desatualizada ${ }^{19}$. Com isso, o objetivo seria beneficiar o país com um sistema de vigilância da fluoretação das águas assentado em informações confiáveis e melhorar a qualidade dos dados disponíveis sobre a cobertura da fluoretação no Brasil20. Neste aspecto, ao compararmos a Portaria em vigência com a nova classificação da concentração de $\mathrm{F}$ em água de abastecimento, verifica-se que a proposta do CECOL/USP leva em conta o desenvolvimento da cárie e fluorose enquanto doenças crônicas e estratificam as faixas de concentração de $\mathrm{F}$ de acordo com diferentes graus de risco. No olhar da vigilância em saúde, esta nova classificação flexibiliza os limites rígidos da concentração de $F$ (mínimo 0,6 ppm F e máximo 0,8 ppm F) da Portaria 635/1975, e propõe uma faixa de concentração, que é coerente considerando simultaneamente o risco e o benefício21. o CECOL/USP propôs ainda o desenvolvimento de um projeto de investigação científica intitulado: Projeto Vigiflúor, que busca desenvolver e aplicar instrumentos para a produção e apuração de dados sobre cobertura e vigilância da fluoretação de águas em municípios de médio e grande porte demográfico 22 .

Segundo os dados obtidos neste trabalho, pode-se notar nesses municípios, a prevalência de poços artesianos como fonte de captação de água na maioria dos municípios. A prevalência de poços justifica-se pela presença de um lençol freático com uma vazão suficiente para atender a demanda da população e que essa água possui qualidade e viabilidade técnica para a sua captação ${ }^{2}$. O município de Sarandi, por exemplo, encontra-se em um desajuste na sua rede de distribuição, devido à ausência de planejamento, dimensionamento da rede, falta de um sistema de produção adequado e reservatórios. A solução para esse município seria realizar um replanejamento de toda a rede de distribuição de água, mantendo primeiramente os poços com boa vazão e perfurar novos poços ou captar água dos mananciais de superfície.

Com relação à frequência com que é feito o controle da concentração de F, oito dos vinte e três municípios com água fluoretada relataram que a companhia de abastecimento faz diariamente o controle do teor de F. Seguindo a Portaria nº 518 de 24 de março de 2004, que normatiza os procedimentos e responsabilidades relativos ao controle e vigilância da qualidade de água para consumo humano, é determinado que o controle dos níveis de $\mathrm{F}$ na água deve ser realizado diariamente ${ }^{2}$. Um controle operacional (realizado pela própria companhia de saneamento) criterioso, utilizando metodologias validadas e padronizadas, poderia ser uma forma econômica e viável de verificação da concentração do fluoreto. Entretanto, isso não eliminaria a necessidade da realização do heterocontrole, ou seja, o controle realizado por instituições externas, para garantir que as avaliações operacionais sejam monitoradas e a água disponibilizada atenda aos critérios de qualidade.

O resultado das análises das águas do presente trabalho mostraram que $79,5 \%$ das amostras de água fluoretada se encontraram na faixa de melhor risco-benefício, 0,55 ppm F a 0,84 ppm F, pela nova classificação proposta pelo CECOL ${ }^{9}$. Este resultado também pode ser percebido no trabalho realizado em Jaguaribara/CE, onde 63,9\% das amostras estavam no melhor risco-benefício?. Dados do heterocontrole de $F$ no município de Bauru/SP, utilizando a classificação proposta pelo CECOL/USP, mostraram que 69\% das amostras se encontravam dentro da faixa de maior benefício do $F$ na prevenção de cárie e menor risco à fluorose ${ }^{23}$. Esses mesmos dados foram avaliados e, considerando a portaria vigente (Portaria 635/1975), foi encontrado que 85\% das amostras estavam dentro dos níveis aceitáveis. Em estudos de heterocontrole realizados nos municípios de Cachoeira do Sul/RS24, Campo Grande/MS25, Pelotas/RS3 e Piracicaba/SP26, os resultados mostraram que as águas de abastecimento público encontravam-se dentro dos níveis aceitáveis. Em 
contrapartida, no estado do Piauí apenas 4,3\% das amostras se encontravam dentro de níveis ideais e a maioria apresentava-se em condições abaixo da faixa ideal27. Todas as amostras de água analisadas em um estudo realizado no município do Rio de Janeiro e 53,57\% das amostras analisadas em São Luís/MA, a concentração de $F$ estava abaixo da faixa ideal, comprometendo os benefícios do processo de fluoretação ${ }^{28}$. Na cidade de Ponta Grossa/PR, dois terços das amostras encontravamse fora do ideal e estes predominavam para valores acima do aceitável ${ }^{29}$.

Dos 30 municípios pertencentes à 15a Regional de Saúde do Paraná, 76\% $(n=23)$ dos municípios apresentam água fluoretada. De acordo com o portal do MS, 95\% da população urbana do estado do Paraná e 85\% da população total do estado recebem água fluoretada. Segundo o Instituto Brasileiro de Geografia e Estatística, 52,9\% dos distritos brasileiros com água tratada realizam a fluoretação ${ }^{30}$. Sendo assim, a classificação proposta pelo CECOL/USP, sob o olhar da vigilância em saúde, torna menos rígidos os limites da concentração de F da Portaria 635/ BSB/1975, que preconiza concentração mínima de 0,6 ppm F e máxima de 0,8 ppm $\mathrm{F}^{20}$. Logo, breves flutuações na concentração de fluoreto na água, abaixo ou acima da faixa de melhor combinação entre os riscos e os benefícios são toleráveis ao longo do tempo de exposição9 .

Em consequência disso, deve ser considerado que concentrações de $\mathrm{F}$ de benefício insignificante ou risco muito alto são toleráveis apenas se ocorrerem eventualmente por um dia ao longo dos meses; concentrações de benefício mínimo ou risco alto são aceitáveis apenas se não se mantiverem constantes por mais do que sete dias ao longo dos meses; e que concentrações de benefício ou risco moderado são toleráveis apenas se não se mantiverem constantes por mais do que 21 dias ao longo de um ano. A constatação, em qualquer aferição dos teores, de risco moderado, alto, ou muito alto deve desencadear ações que alertem o operador para promover os ajustes no sistema? Assim, como as empresas já realizam a avaliação dos teores de $\mathrm{F}$ constantemente, a validação dos métodos de análise realizados por eles torna-se fundamental, já que a identificação das flutuações pela empresa deveria acontecer imediatamente, assim como as correções necessárias.

Quanto aos municípios ( $n=7)$ que não apresentaram fluoretação das águas de abastecimento público, 100\% das amostras apresentaram risco de produzir fluorose e benefício de prevenção da cárie insignificante. Vale ressaltar que nesses municípios a água é proveniente de locais onde não há presença de $\mathrm{F}$ natural. $\mathrm{O}$ estado do Paraná contém teores de $\mathrm{F}$ natural de até 0,96 ppm F, localizada na região Norte, próximos do limite de risco à saúde humana. A origem dessa grande área flúor-anômala ainda não está esclarecida, mas há fortes indicações de que esteja vinculada a fontes geológicas ${ }^{31}$. Portanto, esses sete municípios onde não há fluoretação encontram-se fora das áreas que apresentam $\mathrm{F}$ natural na água.

O heterocontrole faz parte das ações de vigilância em saúde, entretanto, ainda observamos poucos municípios que fazem sistematicamente o monitoramento e a avaliação dos teores de $\mathrm{F}$ das águas de abastecimento público, de modo a realizar intervenções imediatas. Assim, são necessários estudos que verifiquem a realização do heterocontrole como ações da vigilância em saúde. Além disso, vale ressaltar a importância do conhecimento do histórico da fluoretação no município, para possibilitar a avaliação do impacto da fluoretação no controle da cárie dentária e o monitoramento dos possíveis efeitos indesejados ao longo do tempo, por meio dos estudos epidemiológicos.

\section{CONCLUSÃO}

Concluiu-se que na maioria dos municípios pesquisados ocorre a fluoretação da águas de abastecimento, entretanto, o heterocontrole não tem sido realizado. Apesar da maioria das amostras avaliadas apresentarem-se dentro da faixa de concentração de F de melhor combinação risco- 
benefício em relação a saúde bucal, o heterocontrole é necessário para a identificação de flutuações no teor de F, a fim de garantir a qualidade da água à população. Além disso, a classificação das águas propostas pelo CECOL/USP parece ser adequada para a avaliação da fluoretação das águas de abastecimento público.

\section{REFERÊNCIAS}

1. Brasil. Lei $n^{\circ}$ 6050, de 24 de maio de 1974. Dispõe sobre a obrigatoriedade da fluoretação das águas em sistemas de abastecimento. Brasília. Ministério da Saúde; 1974.

2. Brasil. Ministério da Saúde (BR). Diretrizes da política nacional de saúde bucal. Brasília: Ministério da Saúde; 2004.

3. Lima FG. Vinte e quatro meses de heterocontrole da flouretação das águas de abastecimento público de Pelotas, Rio Grande do Sul, Brasil. Cad Saúde Pública. 2004; 20(2): 422-429.

4. Ferreira RGLA, Marques RAA, Menezes, LMB, Narvai PC. Múltiplos aspectos do uso do flúor em saúde pública na visão de lideranças da área de saúde. Ciênc \& Saúde Coletiva. 2013; 18(7): 2139-46.

5. Pinto VG. Saúde bucal coletiva. São Paulo; 2000

6. Cury JA. O Uso do Flúor e o Controle da Cárie como Doença. Odontologia Restauradora - Fundamentos e Possibilidades. São Paulo; 2001.

7. Peixoto DF, Alencar KP, Peixoto RF, Sousa CFM, Sampaio FC, Forte FDS. Heterocontrole da fluoretação da água de abastecimento público do município de Jaguaribara, Ceará. Rev Bras Promoç Saúde. 2012; 25(3): 271-277.

8. Ramires I, Bazulaf MAR. A fluoretação da água de abastecimento público e seus benefícios no controle da cárie dentária - cinqüenta anos no Brasil. Ciênc \& Saúde Coletiva. 2007; 12(4):1057-1065.

9. Centro Colaborador do Ministério da Saúde em Vigilância da Saúde Bucal. Consenso técnico sobre classificação de águas de abastecimento público segundo o teor de flúor. São Paulo: Faculdade de Saúde Pública da Universidade de São Paulo; 2011.

10. Motter J, Moyses ST, França BHS, Carvalho ML, Moysés SJ. Análise da concentração de flúor na água em Curitiba, Brasil: comparação entre técnicas. Rev Panam Salud Publica. 2011; 29(2): 120-125.

11. Zilbovicius C, Ferreira RGLA, Narvai PC. Fluoretação da água e revogação da Lei Federal n. 6.050/1974. Rev Dir Sanit. nov. 2017/ fev.2018; 18(3): 104-124.

12. Frazão $P$, Narvai $P C$. Fluoretação da água em cidades brasileiras na primeira década do século XXI. Rev Saúde Pública 2017:51(47):1-11.

13. Narvai PC. Fluoretação da água: heterocontrole no município de São Paulo no período 1990-1999. Rev Bras Odontol Saúde Coletiva. 2000; 1(2):50-6.

14. Venturini CQ, Narvai PC, Manfredini MA, Frazão P. Vigilância e monitoramento de fluoretos em água de abastecimento público: uma revisão sistemática. Rev Ambient Água. 2016; 11(4).

15. Iheozor-Ejiofor Z, Worthington HV, Walsh T, O'Malley L, Clarkson JE, Macey R, Alam R, Tugwell P. Welch V, Glenny AM. Water fluoridation for the prevention of dental caries. Cochrane Database of Systematic Reviews. 2015; 6: 1-274.
16. McDonagh MS, Whiting PF, Wilson PM, Sutton AJ, Chestnutt I, Cooper Jm Missi K, Bradley M, Treasure E, Kleiinen J. Systematic review of water fluoridation. BMJ. 2000; 321(7265): 855-859.

17 Brasil. Fundação Nacional de Saúde. Manual de saneamento. Brasília: $3^{a}$ edição; 2004.

18. Brasil. Ministério da Saúde. Portaria $n^{\circ}$ 2914, de 12 de dezembro de 2011. Dispõe sobre os procedimentos de controle e de vigilância da qualidade da água para consumo humano e seu padrão de potabilidade. Ministério da Saúde.

19. Faquim J, Soares CCS, Zilbovicius C, Panico C, Narvai PC. Frazão P. CECOL/USP apresenta sugestões na Consulta Pública sobre a Portaria MS 2.914, de 12/12/2011. São Paulo: Centro Colaborador em Vigilância da Saúde Bucal do Ministério da Saúde: 2015 Acesso em 23 nov 2017. Disponível em http://www.cecol.fsp.usp. $\mathrm{br} / \mathrm{dcms} /$ uploads/arquivos/1449682036 CECOL-USP-Sugest\%C 3\%B5esConsultaP\%C3\%BAblicaPortariaMS2914de12dez2011.pdf 20. Bergamo ETP, Barbana M, Terada RSS, Cury JA, Fujimaki M. Fluoride concentrations in the water of Maringá, Brazil, considering the benefit/risk balance of caries and fluorosis. Braz Oral Res. 2015:29(1):1-6.

21. Brasil. Ministério da Saúde. Portaria 635/ Bsb/1975. Estabelece os critérios e teores para adição do íon fluoreto. Brasília: Ministério da Saúde; 1975. Disponível em: http://dtr2004.saude.gov.br/dab/ saudebucal/legislacao/pportaria635 26 12 75.pdf

22. Soares CCS, Simões CAD, Freire MCM, Narvai PC, Frazão P. Pereira PZ, Souza TAC. Projeto Vigifluor - Manual de Credenciamento do Agente do Programa Vigifluor; 2014.

23. Ramires I, Maia LP, Rigolizzo DS, Lauris JRP, Buzalaf MAR Heterocontrole da fuoretação da água de abastecimento público em Bauru, Saõ Paulo, Brasil. Rev Saúde Pública. 2006; 2006 40(5): $883-889$

24. Piva F, Tovo MF, Kramer PF. Heterocontrole da fluoretação da água de abastecimento público do município de Cachoeira do Sul - RS. Rev Fac Odontol. 2006; 47(2): 29-32.

25. Bellé BLL, Lacerda VR, De Carli AD, Zafalon EJ, Pereira PZ Análise da fluoretação da água de abastecimento público da zona urbana do município de Campo Grande (MS). Ciênc \& Saúde Coletiva. 2009: 14(4): 1261-1266.

26. Amaral RC, Wada RS, Souza M da LR de. Concentração de fluoreto nas águas de abastecimento público relacionada à temperatura em Piracicaba - SP. Ver Facul Odontol - UPF. 2007; 12(3): 24-28.

27. Silva JS da, Val CM do, Costa JN, Moura MS de, Silva TAE, Sampaio FC. Heterocontrole da fluoretação das águas em três cidades no Piauí, Brasil. Cad Saúde Pública. 2007; 23(5): $1083-$ 1088.

28. Carmo CDS do, Alves CMC, Cavalcante PR, Ribeiro CCC Avaliação da fluoretação da água de abastacimento público na ilha de São Luís, Maranhão, Brasil. Ciênc \& Saúde Coletiva. 2010: 15 (supl. 1): 1835-1840

29. Wambier DS, Pinto MHB, Kloth AEG, Vetorazzi ML, Ditterich RG, Oliveira DK de. Análise do teor de flúor nas águas de abastecimento público de Ponta Grossa-PR: dez meses de heterocontrole. UEPG Ci Biol Saúde. 2007; 13 (1/2): 65-72.

30. Brasil. Instituto Brasileiro de Geografia e Estatística. Brasília. Ministério do Planejamento, Orçamento e Gestão; 2000.

31. Licht OAB. Atlas geoquímico do Estado do Paraná: Mineropar / Fundação Araucária. Curitiba: 1a edição; 2001. Curitiba, Mineropar / Fundação Araucária.

RECEBIDO : 22/02/2018 